\title{
Lesões Desportivas na Ginástica Artística: Estudo a Partir de Morbidade Referida
}

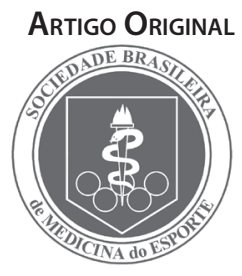

\section{Sports Injuries in Artistic Gymnastics: a Study From Referred Morbidity}

Rosangela Akemi Hoshi ${ }^{1}$ Carlos Marcelo Pastre²

Luiz Carlos Marques Vanderlei ${ }^{2}$ Jayme Netto Júnior ${ }^{2}$

Fábio do Nascimento Bastos ${ }^{1}$

1. Aluno do curso de mestrado em fisioterapia - Faculdade de Ciências e Tecnologia da Universidade Estadual Paulista, campus de Presidente Prudente.

2. Professor doutor do departamento de Fisioterapia Faculdade de Ciências e Tecnologia da Universidade Estadual Paulista, campus de Presidente Prudente.

\section{Endereço para correspondência:}

Faculdade de Ciências e Tecnologia - UNESP

Departamento de Fisioterapia

Rua Roberto Simonsen, no 305,

Presidente Prudente - SP

CEP 19060-900

E-mail: pastre@fct.unesp.br

Submetido em 07/11/2007

Versão final recebida em 07/04/2008

Aceito em 11/04/2008

\begin{abstract}
RESUMO
Introdução: Os níveis de exigência biomecânica devidos ao alto grau de dificuldade na realização de gestos fazem da ginástica artística (GA) uma modalidade com elevado risco de lesões. Assim, é necessário que os aspectos a elas relacionados sejam controlados. Objetivo: Analisar a ocorrência de lesões na Ginástica Artística, associando-as a fatores de risco específicos da modalidade e do atleta, a partir de inquérito de morbidade referida. Métodos: Foram entrevistados 54 ginastas, recrutados ao acaso, classificados segundo o nível competitivo em duas categorias: regional e nacional. Utilizou-se o inquérito de morbidade referida (IMR) com a finalidade de reunir dados sobre a natureza da lesão, região corporal e aparelho ginástico. Os dados foram organizados e apresentados sob a forma de distribuição de freqüências e as variáveis, analisadas segundo nível de associação a partir do teste de Goodman para contrastes entre populações multinomiais, considerando significante o valor $P<0,05$. Resultados: Presença de lesão durante a temporada foi relatada por $39(71,70 \%)$ atletas, sendo $22(56,41 \%)$ mulheres e 17 (43,59\%) homens. Nas categorias regional masculino e feminino e nacional feminino, a maior ocorrência de lesões foi de origem articular, correspondendo a 55,56\%, 50\% e 45,45\% do total, respectivamente. Para o sexo feminino nacional, os membros inferiores foram os mais referidos $(68,18 \%)$ e, em ambas as categorias, as lesões ocorreram nos aparelhos de saltos $(79,41 \%)$, enquanto que no sexo masculino nacional o maior número de agravos foi verificado nos aparelhos de apoio e suspensão (72\%). Conclusões: Há elevada freqüência de lesões, acometendo principalmente articulações e membros inferiores, sendo os aparelhos de saltos os mais referidos quanto à ocorrência de acometimentos. Foi observado também que, quanto maiores as exigências de desempenho técnico, maior a freqüência de lesões.
\end{abstract}

Palavras-chave: traumatismo em atletas, fatores de risco, epidemiologia analítica.

\section{ABSTRACT}

Introduction: The levels of biomechanical demands due to the high level of difficulty in the gestures accomplishment make Artistic Gymnastics (AG) a modality with high risk of injuries. Thus, it is necessary that the aspects concerned with them are controlled. Objective: To analyze the occurrence of injuries in artistic gymnastics, associating them with specific risk factors of the modality and of the athlete from reported morbidity survey. Methods: 54 gymnasts randomly recruited have been interviewed and classified according to their competitive level into two categories: regional and national. The reported morbidity questionnaire (RMQ) was used with the purpose to collect data on the injury's nature, body region and gymnastic apparatus. Data were organized and presented under frequency distribution and analyzed variables. The variables were analyzed according to their level of association from the Goodmann's test for contrasts between multinomials populations considering significant value $p<0.05$. Results: 39 (71.70\%) athletes have reported injury during the season, being 22 of them (56.41) women and 17 (43.59\%) men. In the regional male and female categories and in the national female, the largest reported occurrence of injuries was articular, corresponding to $55.56 \%, 50 \%$ and $45.45 \%$ of the total, respectively. For the national female category, the lower limbs were the most affected (68.18\%). In female, the most frequent injuries occurred in the jumps (79.41\%), while in national male the highest number of complaints has been reported in the support and suspension moves (72\%). Conclusions: There is high frequency of injuries, especially in the joints and lower limbs, being the jump events the most mentioned concerning occurrence. It has also been observed that the higher the level of demand, the higher the occurrence of injuries.

Keywords: athletic injuries, risk factors, analytical studies. 


\section{INTRODUÇÃO}

A ginástica artística (GA) é uma modalidade esportiva que combina arte a gestos biomecânicos de alta complexidade em uma diversidade de eventos. A partir de seu processo evolutivo, observou-se aumento do número de praticantes, elevação do grau de dificuldade dos exercícios e maior exigência do sistema de pontuação, aumentando o risco de lesões ${ }^{(1-7)}$.

Estudos mostram que, nessa modalidade, as lesões são distribuídas pelos tornozelos, joelhos, ombros e coluna lombar, sendo as entorses as mais registradas. Além do aspecto estrutural descrito, os exercícios no solo são os mais propensos à ocorrência de lesões, devido ao alto impacto exercido sobre os segmentos corporais ${ }^{(2,6-9)}$.

Caine e Nassar ${ }^{(7)}$ descrevem que, dentre os fatores intrínsecos responsáveis pela ocorrência de lesões mais freqüentes na ginástica, podem-se destacar estatura, peso e idade. Segundo os autores, maiores valores de estatura e peso predispõem a resultantes de impacto desfavoráveis à integridade das estruturas corporais, incluindo tendões e articulações. Enfatizam a associação entre idade, maior tempo de prática e dificuldade na execução de gestos como fator principal para ocorrência de lesões, não sendo levantados os aspectos biológicos dessa variável.

Sobre os fatores extrínsecos, tem sido investigado o grau de desempenho como causa de lesões. Entretanto, os achados têm-se mostrado inconclusivos. Os dados mostram que quaisquer populações submetidas à prática do esporte em questão são alvo da ocorrência de lesões, independente do nível técnico. Ainda sobre as causas externas, Kirialanis et al.(2) observaram que exercícios no solo representam também um risco, pois são responsáveis por elevado número de lesões agudas dentro da modalidade.

Para as ciências do esporte, o registro de lesões, associadas a seus fatores causais, pode apresentar grande valor profilático e exercer influencia no desempenho(6). Melhoras nas condições de treinamento já foram obtidas por meio de pesquisas realizadas na modalidade, as quais foram responsáveis pela implementação de aparelhos ginásticos. Atualmente, os aparelhos utilizados apresentam maior absorção de impactos e auxiliam a impulsão para realização de movimentos específicos, exigindo menores esforços físicos do ginasta, prevenindo lesões e aumentando performance $e^{(2,8,10,11)}$.

A partir do entendimento sobre o tema, controle, prevenção e reabilitação das lesões são facilitados, o que pode resultar em manutenção da saúde do atleta, retornos mais seguros à prática atlética e melhor desempenho esportivo devido à continuidade do treinamento ${ }^{(12)}$. Portanto, faz-se necessária observação mais detalhada sobre aspectos relacionados aos ginastas e aos riscos de lesões, para contemplar integralmente os objetivos das equipes de saúde ligadas à modalidade.

Assim, constitui-se como objetivo do presente estudo analisar a ocorrência de lesões (freqüência, tipo e segmento anatômico) na ginástica artística, associando-as a fatores de risco específicos da modalidade (aparelhos ginásticos) e do atleta (idade, peso, estatura, tempo de treinamento e categoria), em cada sexo, a partir de inquérito de morbidade referida.

\section{MÉTODOS}

\section{População de estudo}

A coleta de dados foi feita no segundo semestre de 2006, em competições promovidas pela Secretaria Estadual de Esportes de São Paulo e a amostra foi composta por 54 ginastas, idade 15,27 $\pm 4,46$ anos, estatura $1,54 \pm 0,13 \mathrm{~m}$, peso $48 \pm 16,57 \mathrm{~kg}$, tempo de treinamento $6,23 \pm 3,52$ anos, classificados em duas categorias: regional e nacional. Consideraram-se como critérios de inclusão: praticar a modalidade havia mais de um ano, estar treinando ativamente nos últimos três meses e participar de competições durante a temporada corrente.

Optou-se pela seleção dos voluntários ao acaso, durante competições de ginástica artística, os quais tomaram parte do estudo após autorização de seus responsáveis legais, mediante leitura e compreensão de um termo de consentimento livre e esclarecido, não havendo exclusões de qualquer natureza. Cabe destacar que o projeto que envolveu a presente pesquisa foi apreciado e aprovado pelo comitê de ética em pesquisa da Faculdade de Ciências e Tecnologia de Presidente Prudente - Unesp sob protocolo no 096/2006.

\section{Técnicas e procedimentos de campo}

Os dados utilizados para a execução do trabalho foram obtidos por meio de inquérito de morbidade referida (IMR). Os inquéritos são a forma mais adequada de obter informações sobre o estado de saúde de grupos populacionais específicos ${ }^{(13)}$, tendo como objetivo, na presente pesquisa, reunir dados sobre a freqüência, tipo de lesão, região corporal e aparelho ginástico, associados ao treinamento e às competições.

Para realização do levantamento, foi elaborado um questionário específico baseado no inquérito descrito por Pastre et al. ${ }^{(14)}$ e modificado segundo a realidade da modalidade, solicitando informações retroativas à temporada de treinamento, ou seja, aproximadamente 12 meses.

Todo o processo de entrevista foi conduzido pelo pesquisador, desde o questionamento em si, até as anotações no inquérito. Posteriormente, as fichas de coleta foram numeradas para facilitar o registro das informações e os dados foram registrados em planiIha computacional Excel, para organização, sistematização e análise dos dados.

\section{Descrição do inquérito de morbidade (apêndice 1)}

O instrumento apresenta campo para preenchimento de informações pessoais dos atletas como sexo, idade, peso, estatura e tempo de treinamento em anos. Em se tratando de lesões, os atletas responderam a respeito do tipo de agravo, local anatômico e aparelho ginástico em que as lesões ocorreram.

Para efeito de estudo considerou-se lesão desportiva (LD) qualquer dor ou afecção musculoesquelética resultante de treinamentos e/ou competições desportivas, suficiente para causar alterações no treinamento normal, seja na forma, duração, intensidade ou freqüência, conforme já utilizado em outras pesquisas(6,14-16).

Para fins de estratificação, foi questionado em que tipos de competições o atleta participava, classificando a categoria (regional ou nacional) em que se enquadrava, homogeneizando a população investigada e direcionando a análise dos dados. Consideraram-se de categoria regional os atletas que realizavam competições de menor expressão, como torneios internos, chegando ao máximo a disputas dentro da região onde treinam. Para ser considerado de categoria nacional, os atletas deveriam participar de eventos de maior expressão, como campeonatos interestaduais ou nacionais.

\section{Organização e descrição das categorias das variáveis}

Para facilitar a análise e apresentação dos resultados, as categorias ou subdivisões das variáveis, já descritas, foram agrupadas, sem, 
no entanto, modificar a essência de sua origem ou as conclusões do estudo.

Inicialmente, é destacada a variável aparelho ginástico. Entende-se como "aparelhos de apoio e suspensão" o conjunto que compreende as barras paralelas assimétricas, barras paralelas simétricas, barra fixa, cavalo com alças e argolas. Os aparelhos solo, salto e trave foram agrupados na categoria "aparelhos de saltos".

Para a variável tipo de lesão, foram organizadas quatro tipos de categorias: lesão muscular (distensão, contratura, mialgia), tendinopatias (tendinite, lesão tendínea), lesão articular (luxação, sinovite, entorse, lesão condral) e lesão óssea (fraturas e periostite). Dessa maneira, desde que se enquadrasse na definição de LD utilizada, as lesões foram distribuídas nos grupos.

O local anatômico foi classificado em três partes: membros superiores, membros inferiores e tronco, considerando qualquer região em todo o complexo dos segmentos citados.

\section{Procedimentos estatísticos}

A comparação de ocorrência de lesão e de sexo nas variáveis antropométricas e de treinamento dos atletas foi realizada pelo teste $t$ de Student para amostras independentes ou pelo teste não paramétrico de Mann-Whitney. A opção quanto ao tipo de teste foi realizada sob a aderência dos dados à distribuição normal de probabilidade. As variáveis peso e estatura responderam como aderentes e, as demais (idade e anos de treinamento) receberam no procedimento estatístico a opção não-paramétrica.

O estudo de prevalência de lesões em cada grupo (categoria e sexo) foi feito por meio de distribuição de freqüências (absoluta e relativa) e por taxas de lesão por atleta e por atletas lesionados.

Para o estudo da associação entre tipo de lesão, local anatômico e aparelho ginástico, utilizou-se o teste de Goodman para contrastes entre e dentro de populações multinomiais.

\section{RESULTADOS}

Dentre os 54 participantes, 39 (72,22\%) relataram lesão desportiva e observou-se a presença de 1,26 lesão por participante. Destaca-se ainda que, para as variáveis idade, peso, estatura e tempo de treinamento, não foram observadas diferenças estatisticamente significantes dentro de cada categoria e sexo, quando comparados os valores descritivos (média ou mediana) dos grupos com e sem presença de lesão $(P>0,05)$.

Observa-se na tabela 1 que as variáveis antropométricas apresentam diferenças significantes entre os sexos $(P<0,05)$ e que há tendência de relação entre maiores valores para idade e categoria nacional em ambos os sexos, o que acontece também para a variável anos de treinamento. Não há diferença entre altura e peso no sexo feminino entre as categorias, ao contrário do que acontece no masculino.

A tabela 2 mostra que as taxas de lesão são maiores para os homens quando comparados com as mulheres e maiores para a categoria nacional quando comparada com a regional. A distribuição de freqüências dentro de cada sexo também é maior na categoria nacional.

Observa-se, na tabela 3, que no sexo masculino as lesões articulares apresentam freqüências relativas maiores na categoria regional quando comparada com a nacional $(P<0,05)$. Para o sexo feminino, as lesões articulares também foram as mais relatadas, mas nesse caso em ambas as categorias.

Não houve diferença significante tanto para os homens quanto para as mulheres, entre as categorias, quando se analisou a distribuição
Tabela 1. Medidas descritivas das variáveis antropométricas e de treinamento de acordo com o sexo e a categoria

\begin{tabular}{|c|c|c|c|c|c|}
\hline \multirow{3}{*}{ Variáveis } & \multirow{3}{*}{ Sexo } & \multicolumn{4}{|c|}{ Categoria } \\
\hline & & \multicolumn{2}{|c|}{ Regional $(n=25)$} & \multicolumn{2}{|c|}{ Nacional $(n=29)$} \\
\hline & & Média & Mediana & Média & Mediana \\
\hline \multirow{2}{*}{$\begin{array}{l}{ }^{2} \text { Idade } \\
\text { (anos) }\end{array}$} & Feminino & $12,05 \pm 2,3$ & $\begin{array}{c}12 \mathrm{a} \\
\mathrm{A}\end{array}$ & $13,75 \pm 2,049$ & $\begin{array}{c}13,5 \mathrm{a} \\
\mathrm{B}\end{array}$ \\
\hline & Masculino & $16,37 \pm 5,09$ & $\begin{array}{c}15 b \\
A\end{array}$ & $20,69 \pm 3,19$ & $\begin{array}{c}21 b \\
B\end{array}$ \\
\hline \multirow{2}{*}{$\begin{array}{l}{ }^{1} \text { Altura } \\
\text { (metros) }\end{array}$} & Feminino & $\begin{array}{c}1,45 \pm 0,10 \mathrm{a} \\
\mathrm{A}\end{array}$ & 1,44 & $\begin{array}{c}1,5 \pm 0,06 \mathrm{a} \\
\mathrm{A}\end{array}$ & 1,51 \\
\hline & Masculino & $\begin{array}{c}1,59 \pm 0,11 b \\
A\end{array}$ & 1,59 & $\begin{array}{c}1,70 \pm 0,07 b \\
B\end{array}$ & 1,72 \\
\hline \multirow{2}{*}{$\begin{array}{c}\text { 'Peso } \\
\text { (quilograma) }\end{array}$} & Feminino & $\begin{array}{c}37,23 \pm 11,98 \mathrm{a} \\
\mathrm{A}\end{array}$ & 32,5 & $\begin{array}{c}41,28 \pm 6,78 \mathrm{a} \\
\mathrm{A}\end{array}$ & 41,1 \\
\hline & Masculino & $\begin{array}{c}54,67 \pm 14,98 b \\
A\end{array}$ & 56,6 & $\begin{array}{c}70,0 \pm 7,59 b \\
B\end{array}$ & 70,4 \\
\hline \multirow{2}{*}{$\begin{array}{l}{ }^{2} \text { Anos de } \\
\text { treinamento }\end{array}$} & Feminino & $3,85 \pm 2,28$ & $\begin{array}{c}3 a \\
A\end{array}$ & $6,37 \pm 1,76$ & $\begin{array}{c}6 \mathrm{a} \\
\mathrm{B}\end{array}$ \\
\hline & Masculino & $4,12 \pm 1,95$ & $\begin{array}{c}4,5 \mathrm{a} \\
\mathrm{A}\end{array}$ & $10,46 \pm 3,40$ & $\begin{array}{c}12 b \\
B\end{array}$ \\
\hline \multirow{2}{*}{$\begin{array}{c}\mathrm{N}^{\circ} \text { de } \\
\text { indivíduos }\end{array}$} & Feminino & \multicolumn{2}{|l|}{17} & \multicolumn{2}{|l|}{16} \\
\hline & Masculino & \multicolumn{2}{|l|}{8} & \multicolumn{2}{|l|}{13} \\
\hline
\end{tabular}

'Resultados do teste estatístico para média e desvio-padrão da variável estudada; Teste t de Student para amostras independentes (peso e estatura): ${ }^{2}$ Resultado do teste estatístico para mediana da variável estudada; Teste não paramétrico de Mann-Whitney (idade e anos de treinamento).

Tabela 2. Distribuição dos atletas lesionados, lesão relatada, taxa de lesão por atleta e taxa de lesão por atleta lesionado segundo sexo e categoria

\begin{tabular}{|c|c|c|c|c|}
\hline \multirow[b]{2}{*}{ Variáveis } & \multirow[b]{2}{*}{ Sexo } & \multicolumn{2}{|c|}{ Categoria } & \multirow[b]{2}{*}{ Total } \\
\hline & & $\begin{array}{l}\text { Regional } \\
(n=14)\end{array}$ & $\begin{array}{l}\text { Nacional } \\
(n=25)\end{array}$ & \\
\hline \multirow{2}{*}{ Atletas lesionados } & Feminino & $9(40,90 \%)$ & $13(59,10 \%)$ & $22(100 \%)$ \\
\hline & Masculino & $5(29,41 \%)$ & $12(70,59 \%)$ & 17 (100\%) \\
\hline \multirow{2}{*}{ Lesões relatadas } & Feminino & $12(35,30 \%)$ & $22(64,70 \%)$ & $34(100 \%)$ \\
\hline & Masculino & $9(26,47 \%)$ & $25(73,53 \%)$ & $34(100 \%)$ \\
\hline \multirow{2}{*}{$\begin{array}{c}\text { Taxa de lesão por } \\
\text { atleta }\end{array}$} & Feminino & 0,70 & 1.38 & 1,03 \\
\hline & Masculino & 1,12 & 1,92 & 1,61 \\
\hline \multirow{2}{*}{$\begin{array}{l}\text { Taxa de lesão por } \\
\text { atleta lesionado }\end{array}$} & Feminino & 1,33 & 1,69 & 1,55 \\
\hline & Masculino & 1,8 & 2,08 & 2 \\
\hline
\end{tabular}

Nota: A taxa de lesão por atleta foi obtida pela divisão do número total de lesões pelo número total de atletas entrevistados e a taxa de lesão por atleta acometido é igual ao número total de lesões dividido pelo total de atletas lesionados.

das lesões segundo local anatômico. Para os homens, na categoria regional, os membros inferiores são os mais acometidos e na nacional, os membros superiores. No sexo feminino, a maior freqüência fica para o acometimento dos membros inferiores na categoria nacional $(P<$ 0,05), conforme observado na tabela 4. 
Tabela 3. Distribuição da variável tipo de lesão de acordo com o sexo e a categoria.

\begin{tabular}{|c|c|c|c|c|c|c|}
\hline \multirow[b]{2}{*}{ Sexo } & \multirow[b]{2}{*}{ Categoria } & \multicolumn{5}{|c|}{ Tipo de lesão } \\
\hline & & Muscular & Articular & Óssea & $\begin{array}{c}\text { Tendino- } \\
\text { patia }\end{array}$ & Total \\
\hline \multirow{2}{*}{ Masculino } & Regional & $\begin{array}{c}1(11,11) a \\
A\end{array}$ & $\begin{array}{c}5(55,56) b \\
B\end{array}$ & $\begin{array}{c}3(33,33) a \\
A\end{array}$ & $\begin{array}{c}0(0,00) \text { a } \\
\text { A }\end{array}$ & $9(100)$ \\
\hline & Nacional & $\begin{array}{c}3(12,00) a \\
A\end{array}$ & $\begin{array}{c}7(28,00) a \\
A\end{array}$ & $\begin{array}{c}11(44,00) \mathrm{a} \\
\mathrm{A}\end{array}$ & $\begin{array}{c}4(16,00) \mathrm{a} \\
\mathrm{A}\end{array}$ & $25(100)$ \\
\hline \multicolumn{2}{|c|}{ Total } & $4(11,76)$ & $12(35,30)$ & $14(41,18)$ & $4(11,76)$ & $34(100)$ \\
\hline \multirow{2}{*}{ Feminino } & Regional & $\begin{array}{c}3(25,00) a \\
A\end{array}$ & $\begin{array}{c}6(50,00) a \\
B\end{array}$ & $\begin{array}{c}3(25,00) \mathrm{a} \\
\mathrm{A}\end{array}$ & $\begin{array}{c}0(0,00) \text { a } \\
\text { A }\end{array}$ & $12(100)$ \\
\hline & Nacional & $\begin{array}{c}6(27,27) a \\
A\end{array}$ & $\begin{array}{c}10(45,45) \mathrm{a} \\
\mathrm{B}\end{array}$ & $\begin{array}{c}5(22,73) \mathrm{a} \\
\mathrm{A}\end{array}$ & $\begin{array}{c}1(4,55) \mathrm{a} \\
\mathrm{A}\end{array}$ & $22(100)$ \\
\hline \multicolumn{2}{|c|}{ Total } & $9(26,47)$ & $16(47,06)$ & $8(23,53)$ & $1(2,94)$ & $34(100)$ \\
\hline
\end{tabular}

Teste de Goodman para contrastes entre e dentro de populações multinomiais, onde as letras maiúsculas são para relacionar as linhas, sendo $A<B$ e as letras minúsculas, as colunas, sendo $a<b$; Os valores entre parênteses são as freqüências relativas de cada valor.

Tabela 4. Distribuição da variável local anatômico da lesão de acordo com o sexo e a categoria

\begin{tabular}{|c|c|c|c|c|c|}
\hline \multirow{2}{*}{ Sexo } & \multirow{2}{*}{ Categoria } & \multicolumn{4}{|c|}{ Local anatômico } \\
\hline & & MMSS & Tronco & MMII & Total \\
\hline \multirow{2}{*}{ Masculino } & Regional & $\begin{array}{c}4(44,44) a \\
B\end{array}$ & $\begin{array}{c}0(0,00) \text { a } \\
A\end{array}$ & $\begin{array}{c}5(56,66) a \\
B\end{array}$ & $9(100)$ \\
\hline & Nacional & $\begin{array}{c}16(64,00) \mathrm{a} \\
\mathrm{B}\end{array}$ & $\begin{array}{c}2(8,00) a \\
A\end{array}$ & $\begin{array}{c}7(28,00) a \\
A B\end{array}$ & $25(100)$ \\
\hline \multicolumn{2}{|c|}{ Total } & $20(58,82)$ & $2(5,82)$ & $12(35,29)$ & $34(100)$ \\
\hline \multirow{2}{*}{ Feminino } & Regional & $\begin{array}{c}5(41,67) a \\
A\end{array}$ & $\begin{array}{c}1(8,33) \mathrm{a} \\
\mathrm{A}\end{array}$ & $\begin{array}{c}6(50,00) a \\
A\end{array}$ & $12(100)$ \\
\hline & Nacional & $\begin{array}{c}3(13,64) a \\
A\end{array}$ & $\begin{array}{c}4(18,18) a \\
A\end{array}$ & $\begin{array}{c}15(68,18) a \\
B\end{array}$ & $22(100)$ \\
\hline \multicolumn{2}{|c|}{ Total } & $8(23,53)$ & $5(14,71)$ & $21(61,76)$ & 34 (100) \\
\hline
\end{tabular}

Teste de Goodman para contrastes entre e dentro de populações multinomiais, onde as letras maiúsculas são para relacionar as linhas, sendo $A<B$ e as letras minúsculas, as colunas, sendo $a<b$. Os valores entre parênteses são as freqüências relativas de cada valor.

Para os homens, a maior freqüência de lesões ocorreu para categoria nacional realizando exercícios em aparelhos de apoio e suspensão $(P<0,05)$ em relação à categoria regional e aos aparelhos de saltos. No feminino, os aparelhos de saltos apresentaram maior freqüência em ambas as categorias (tabela 5).

\section{DISCUSSÃO}

A obtenção de dados a respeito das modalidades esportivas e seus fatores de risco é de grande importância para as Ciências do Esporte, uma vez que pode gerar subsídios para prevenção de lesões, bem como proporcionar retorno precoce do atleta às suas rotinas. Além disso, oferece suporte ao desenvolvimento de novas técnicas e
Tabela 5. Distribuição de ocorrência de lesões nos aparelhos ginásticos, segundo o sexo e a categoria

\begin{tabular}{|c|c|c|c|c|}
\hline \multirow{2}{*}{ Sexo } & \multirow{2}{*}{ Categoria } & \multicolumn{3}{|c|}{ Aparelho ginástico } \\
\hline & & De saltos & De apoio e suspensão & Total \\
\hline \multirow{2}{*}{ Masculino } & Regional & $\begin{array}{c}6(66,67) b \\
A\end{array}$ & $\begin{array}{c}3(33,33) \mathrm{a} \\
\mathrm{A}\end{array}$ & $9(100)$ \\
\hline & Nacional & $\begin{array}{c}7(28,00) \mathrm{a} \\
\mathrm{A}\end{array}$ & $\begin{array}{c}18(72,00) b \\
B\end{array}$ & $25(100)$ \\
\hline \multicolumn{2}{|c|}{ Total } & $13(38,24)$ & $21(61,76)$ & $34(100)$ \\
\hline \multirow{2}{*}{ Feminino } & Regional & $\begin{array}{c}10(83,33) \mathrm{a} \\
\mathrm{B}\end{array}$ & $\begin{array}{c}2(16,77) a \\
A\end{array}$ & $12(100)$ \\
\hline & Nacional & $\begin{array}{c}17(77,27) a \\
B\end{array}$ & $\begin{array}{c}5(22,73) \mathrm{a} \\
\mathrm{A}\end{array}$ & $22(100)$ \\
\hline \multicolumn{2}{|c|}{ Total } & $27(79,41)$ & $7(20,59)$ & $34(100)$ \\
\hline
\end{tabular}

Teste de Goodman para contrastes entre e dentro de populações multinomiais, onde as letras maiúsculas são para relacionar as linhas, sendo $A<B$ e as letras minúsculas, as colunas, sendo $a<b$. Os valores entre parênteses são as freqüências relativas de cada valor.

aparelhagem, visando à qualidade de treinamento(10,11). Desse modo, entendeu-se como apropriada a realização deste estudo.

Realizar levantamento de informações epidemiológicas requer, para efeitos de comparações, implantação de recursos e conceitos similares aos de outras pesquisas. Nesse sentido, na metodologia do presente estudo foi utilizada a definição de LD mais encontrada nos textos da literatura consultada, conforme descrito por Kolt e Kirkby ${ }^{(6)}$, Pastre et al. ${ }^{(12)}$, Grego et al. ${ }^{(15)}$ e Sands et al.(16)

Em relação ao inquérito aplicado, destaca-se sua validação realizada por Pastre et al. ${ }^{(14)}$, em indivíduos da elite do atletismo brasileiro, solicitando informações retroativas à temporada corrente e, então, adaptado para a ginástica artística. A aplicabilidade dessa ferramenta foi satisfatória e os resultados obtidos sugerem boa aderência da mesma na modalidade em questão.

Como principal limitação do estudo, destaca-se a necessidade de agrupamento das categorias das variáveis para facilitar os procedimentos de análise. Assim, detalhes sobre local exato e lesões específicas não são destacados de maneira direta. Portanto, os resultados são extrapolados a partir de características mais amplas de grupos de variáveis mais genéricas. Deve-se enfatizar, entretanto, que tal modelo tem sido adotado em estudos de mesma natureza para melhor aproveitamento dos dados coletados no âmbito esportivo ${ }^{(12-15)}$. Além dessa limitação, ressalta-se a ausência de associação entre lesões e horas de treino e competições, condição importante para verificação de demanda de trabalho exercido pelo atleta.

Os resultados refletem o alto risco de ocorrência de lesões considerando a modalidade. A categoria nacional apresentou o maior número, condição esta já observada por Daly et al. ${ }^{(1)}$, Dixon e Fricker ${ }^{(4)}$ e Meeusen e Borms ${ }^{(17)}$, os quais relataram que o risco de lesões está intimamente relacionado com a categoria, uma vez que, quanto mais elevada a categoria, maior sua exposição aos esforços musculoesqueléticos durante treinamentos e/ou competições.

Kolt e Kirkby ${ }^{(6)}$, que registraram taxa de 3,64 lesões por ginasta em um ano, também observaram maiores valores nos atletas de elite 
em comparação com os da subelite da equipe australiana, os quais, segundo os autores, estão relacionados com as dificuldades dos movimentos executados pelos diferentes níveis de performance. Atletas de alto nível realizam rotinas com maior grau de complexidade, além de possuir maior volume de treinamento.

A elevada freqüência de lesões pode estar relacionada com a baixa faixa etária dos participantes, pressupondo início precoce no esporte. Daly et al. ${ }^{(1)}$ destacam tendência mais elevada à ocorrência de LD nos que se iniciam mais precocemente em esportes com alta complexidade motora, explicado pelo fato de que há deficiência coordenativa, estrutural e de concentração do jovem atleta para desempenhar determinados movimentos, além da falta de experiência para desempenhar certas atividades. Meeusen e Borms ${ }^{(17)}$ mencionam, ainda, que a combinação de períodos de rápido crescimento e intenso treinamento proporcionam condições em que o ginasta é mais propenso à ocorrência de lesões.

Quando separados os voluntários que relataram lesões dos que não o fizeram, notou-se homogeneidade dos valores descritivos de idade, peso, estatura e tempo de treinamento e, nesse sentido, não foram constatadas associações entre tais variáveis e a ocorrência de acometimentos osteomusculares. Esses achados discordam das afirmações de Caine e Nassar ${ }^{(7)}$, que as classificam como fator de risco dentro da modalidade em questão.

Sobre o tipo de agravo, o mais registrado neste estudo foi de natureza articular em ambos os sexos, como também verificado por Rego et al.(18). Keith et al. ${ }^{(3)}$ observaram que entorses são comuns entre ginastas, devido às altas cargas de impacto exercidas pelas acrobacias, executadas com grande potência, altura e compostas por movimentos rotacionais, como também mencionado por Marshall et al. ${ }^{(19)}$. Além disso, Forkin et al. ${ }^{(20)}$ descrevem que a força de reação do solo é da ordem de oito a 18 vezes o peso corporal no momento da aterrissagem. Nesses eventos, como explicado por Souza e Almeida(21), o impacto sobre estruturas articulares é maior que a capacidade de absorção dos músculos, agredindo o tecido que as compõe.

Sob uma visão geral, o sexo masculino da categoria nacional apresentou a maioria das lesões nos membros superiores, diferente das mulheres, que foram acometidas com maior freqüência nos membros inferiores. Tais achados são similares aos de Dixon e Fricker ${ }^{(4)}$ para o sexo feminino, que consideraram membros inferiores, superiores e tronco, respectivamente, como locais mais referidos.

Os homens da categoria nacional referiram mais lesões nos aparelhos de apoio e suspensão, contrariamente ao observado no grupo feminino, que referiu mais lesões nos membros inferiores e nos aparelhos de saltos. Segundo Caine e Nassar ${ }^{(7)}$, essa diferença ocorre pela natureza dos aparelhos executados por homens, que exigem grande demanda física do tronco superior, o que não ocorre entre as mulheres.

Tais achados corroboram as afirmações feitas por Kirialanis et al. ${ }^{(2)}$, que, além do salto e solo, comum aos dois sexos, citam as barras paralelas como maiores causadoras de lesões nos homens e a trave de equilíbrio, nas mulheres. Esses eventos ocorrem devido ao tipo de sobrecarga biomecânica exercida pelos aparelhos sobre as estruturas. Yeadon e Brewin (22) descrevem, nas argolas, considerado aparelho de apoio e suspensão, neste estudo, a importância da execução de giros e exercícios estáticos, que requerem grande força de musculatura superior para suportar o peso corporal e manter o máximo de alinhamento entre quadril e ombros, o que aumenta a possibilidade de lesões nos membros superiores.

Outro aparelho dessa mesma classe é a barra fixa, onde os atletas realizam uma rotina dinâmica composta basicamente por giros com o corpo todo em extensão. Heley e Yeadon ${ }^{(23)}$ mencionam a necessidade da manutenção de momento angular ideal entre ombros e quadris durante a realização desses movimentos, pois precedem execuções importantes da seqüência, como largadas, retomadas e saídas do aparelho. Assim, segundo Arampatzis e Bruggemann(24), ocorre grande acúmulo de energia potencial e eventos inesperados podem potencializar lesões nas estruturas mais envolvidas durante essas rotinas, ou seja, membros superiores.

Aparelhos ginásticos que possuem como característica a realização de saltos e acrobacias proporcionam cargas principalmente sobre a parte inferior do corpo. Como exemplo, pode ser citado o solo, definido por Rasquinha et al. ${ }^{(25)}$ como um dos mais complexos aparelhos, composto por elementos dinâmicos desenvolvidos pelos ginastas em uma superfície elástica, cujas forças externas atuantes sobre um ginasta durante a execução de seus movimentos podem chegar à ordem de 5 a 17,5 vezes o peso do seu corpo.

O salto sobre o cavalo ou sobre a mesa possui também características de aparelhos de saltos. Nesse, o ginasta deve realizar duas fases, sendo uma antes de chegar com as mãos à superfície do aparelho e outra, após o impulso proporcionado pelos membros superiores empurrando o aparelho. Feito isso, o ginasta atinge certa altura e pode realizar outros elementos de sua rotina, como acrobacias, por exemplo. Muitos desses movimentos são realizados "às cegas", segundo Takei et al.(26), e como o indivíduo não olha onde ou como está aterrissando, o risco de lesões é muito elevado nesse aparelho, sendo os membros inferiores os mais atingidos.

A exploração das lesões e seus fatores de risco revelaram relações importantes envolvendo categorias e sexo, associadas a aparelho ginástico, local anatômico e natureza da lesão. Espera-se que tais informações possam contribuir para melhor entendimento sobre lesões em praticantes dessa modalidade, incluindo formulação e adequação de programas de prevenção e reabilitação, em quaisquer âmbitos de prática.

\section{CONCLUSÕES}

Houve elevada freqüência de lesões na população analisada, com maiores taxas no sexo masculino. Sobre o tipo de lesão, no sexo feminino em ambas as categorias e na categoria regional para os homens, as articulares foram as mais presentes. Em relação ao segmento anatômico, para os homens na categoria regional os membros inferiores são os mais acometidos e, na nacional, os membros superiores; no sexo feminino nacional, a maior freqüência fica para o acometimento dos membros inferiores. Sobre o aparelho ginástico, os de saltos foram os mais referidos pelas mulheres e os de apoio e suspensão, pelos homens da categoria nacional. Por fim, não se constatou associação entre presença de lesão e variáveis antropométricas e tempo de treinamento dos participantes.

Todos os autores declararam não haver qualquer potencial conflito de interesses referente a este artigo. 
Apêndice 1

IMR- Inquérito de morbidade referida

\begin{tabular}{|c|c|c|c|c|c|c|c|c|c|}
\hline \multirow{2}{*}{\multicolumn{10}{|c|}{$\begin{array}{ll}\text { Numero da ficha: } \_ \text {Sexo: } & \text { Idade: } \\
\text { Presença de lesão desportiva na temporada: } \operatorname{Sim}(\text { ) Não ( ) }\end{array}$}} \\
\hline & & & & & & & & & \\
\hline \multicolumn{10}{|c|}{ Características da lesão } \\
\hline \multicolumn{2}{|c|}{ Variáveis } & \multicolumn{8}{|c|}{ Lesões desportivas } \\
\hline \multicolumn{2}{|c|}{ Identificação da lesão desportiva } & $1^{\mathrm{a}}$ & $2^{\mathrm{a}}$ & $3^{a}$ & $4^{a}$ & $5^{\mathrm{a}}$ & $6^{a}$ & $7^{\mathrm{a}}$ & $8^{\mathrm{a}}$ \\
\hline \multicolumn{10}{|l|}{ Tipo de lesão } \\
\hline \multicolumn{10}{|l|}{ Local anatômico } \\
\hline \multicolumn{10}{|l|}{ Aparelho ginástico } \\
\hline \multicolumn{10}{|c|}{ Mecanismo de lesão ou aumento do sintoma } \\
\hline \multicolumn{2}{|l|}{ Momento } & & & & & & & & \\
\hline \multicolumn{10}{|c|}{ Codificação das variáveis } \\
\hline Tipo de lesão & Mecanismo de lesão & \multicolumn{3}{|c|}{ Aparelho ginástico } & \multicolumn{5}{|c|}{ Localização anatômica } \\
\hline 1 - Distensão muscular & 1- Acrobacia & \multicolumn{3}{|l|}{ 1-Solo } & \multicolumn{2}{|c|}{ 1- Ombro } & \multicolumn{3}{|c|}{13 - Coxa posterior } \\
\hline 2 - Contratura muscular & 2- Salto ginástico & \multicolumn{3}{|l|}{ 2- Salto } & \multicolumn{2}{|c|}{2 - Braço } & \multicolumn{3}{|c|}{14 - Joelho } \\
\hline 3 - Tendinopatia & 3 - Giro & \multicolumn{3}{|c|}{3 - Barras paralelas assimétricas } & \multicolumn{2}{|c|}{3 - Antebraço } & \multicolumn{3}{|c|}{15 - Perna } \\
\hline 4 - Entorse & 4- Elemento ginástico & \multicolumn{3}{|c|}{4 - Barras paralelas } & \multicolumn{2}{|c|}{4 - Cotovelo } & \multicolumn{3}{|c|}{16 - Panturrilha } \\
\hline 5 - Mialgia & 5- Parada brusca & \multicolumn{3}{|c|}{5 - Barra fixa } & \multicolumn{2}{|c|}{5 - Punho } & \multicolumn{3}{|c|}{17 - Tornozelo } \\
\hline 6 - Periostite & 6 - Queda & \multicolumn{3}{|c|}{6 - Trave de equilíbrio } & \multicolumn{2}{|c|}{6 - Mão } & \multicolumn{3}{|c|}{18 - Pé } \\
\hline 7 - Sinovite & 7- Trabalho de força & 7 - Argo & & & 7 - Tór & & 14 & Outra & \\
\hline 8 - Fratura & 8 - Alongamento & 8 - Caval & $m$ alças & & $8-A b$ & & & & \\
\hline 9 - Bursite & 9 - Sobrecarga & 9 - Outro & & & $9-\operatorname{Re}$ & lombar & & & \\
\hline 10 - Dor aguda inespecífica & 10 - Corrida & & & & $10-R$ & o cervical & & & \\
\hline 11- Dor crônica inespecífica & 11 - Coreografia & & & & $11-Q$ & & & & \\
\hline 12 - Outra & 12 - Aterrissagem & & & & $12-C$ & anterior & & & \\
\hline & 13 - Outro & & & & $13-0$ & & & & \\
\hline & nto da lesão & & & & & & & & \\
\hline 1 - Treinamento & 2 - Competição & & & & & & & & \\
\hline
\end{tabular}

\section{REFERÊNCIAS BIBLIOGRÁFICAS}

1. Daly RM, Bass SL, Finch CF. Balancing the risk of injury to gymnasts: how effective are the counter measures? Br J Sports Med 2001;35:8-19.

2. Kirialanis $P$, Malliou P, Beneka A, Giannakopoulos K. Ocurrence of acute lower limb injuries in relation to event and exercise phase. $\mathrm{Br} J$ Sports Med 2003;37:137-9.

3. Keith WR, Quinney HA, Clark BH, Douglas H. Knee muscle strength in elite male gymnasts. J Orthop Sports Phys Ther 1995;22:10-7.

4. Dixon M, Fricker P. Injuries to elite gymnasts over 10 yr. Med Sci Sports Exerc 1993;25:1322-9.

5. Foster JB. Efforts to reduce gymnastics injuries focus on spring floor. Biomechanics (Rockville) 2007;14:11-2

6. Kolt GS, Kirkby RJ. Epidemiology of injury in elite an subelite female gymnasts: a comparison of retrospective and prospective findings. Br J Sports Med 1999;33:312-8.

7. Caine D, Nassar L. Espidemiolgy of pediatric sports injuries. Individual sports. Med Sport Sci, Baser, Karger 2005;48:18-58.

8. Eric S. Graceful, beautiful and perilous: as gymnastics routines grow ever trickier, experts worry that children are being pushed beyond their limits and are paying their health. Science 2004;305(5684):641.

9. Harringe ML, Renström P, Werner S. Injury incidence, mechanism and diagnosis in top-level teamgym: a prospective study conducted over one season. Scand J Med Sci Sports 2007;17:115-9.

10. Sano S, Ikegami Y, Hiroyuki N, Apriantomo T, Sakurai S. The continuous measurement of the springboard reaction force in gymnastic vaulting. J Sports Sci 2007;25:381-91.

11. Pain MTG, Miels $C L$, Yeadon MR. Video analysis of the deformation and effective mass of gymnastics landings mats. Med Sci Sports Exer 2005;37(10):1754-60.

12. Pastre CM, Carvalho Filho G, Monteiro HL, Netto Júnior J, Padovani CR. Lesões desportivas na elite do atletismo brasileiro: estudo a partir de morbidade referida. Rev Bras Med Esporte 2005;11(1):43-7.

13. Conte M, Matiello Jr E, Chalita LVS, Gonçalves A. Exploração de fatores de risco de lesões desportivas entre universitários de educação física: estudo a partir de estudantes de Sorocaba/ Sp. Rev Bras Med Esporte 2002;8:151-6.
14. Pastre CM, Carvalho Filho G, Monteiro HL, Netto Júnior J, Padovani CR. Lesões desportivas no atletismo: comparação entre informações obtidas em prontuários e Inquéritos de Morbidade Referida. Rev Bras Med Esporte 2004;10:1-8.

15. Grego LG, Monteiro HL, Padovani R, Gonçalves A. Lesões na dança: estudo transversal híbrido em academias da cidade de Bauru/SP. Rev Bras Med Esporte 1999;5:47-54

16. Sands WA, Shultz BB, Newmann AO. Women's gymnastics injuries. Am J Sports Med 1993;21:271-6.

17. Meeusen R, Borms J. Gymnastic injuries. Sports Med 1992;13:337-56.

18. Rego F, Reis M, Oliveira R. Lesões em Ginastas portugueses de competição das modalidades de Trampolins, Ginástica Acrobática, Ginástica Artística, e Ginástica Rítmica na Época 2005/2006. Rev Port de Fisiot Desporto 2007;1:21-7.

19. Marshall SW, Covassin T, Dick R, Nassar LG, Agel J. Injury incidence, mechanism and diagnosis in top-level teamgym: a prospective study conducted over one season. Scand J Med Sci Sports 2007;17:115-9.

20. Forkin DM, Koczur C, Battle R, Newton RA. Evaluation of kinesthetic Deficits indicative of balance control in gymnasts with unilateral chronic ankle sprains. J Orthop Sports Phys Ther 1996;23:245-50.

21. Souza GM, Almeida FS. Queixa de dor músculo-esquelética dos atletas de 6-20 anos praticantes de Ginástica Artística feminino. Arq Med ABC 2006;31:67-72.

22. Yeadon MR, Brewin MA. Optimized performance of the backward long swing on rings. J Biomech 2003;36:545-52.

23. Heley MJ, Yeadon. Maximal dismounts from high bar. J Biomech 2005;38:2221-7.

24. Arampatzis A, Bruggemann GP. Mechanical energetic process during the giant swing before the tkatchev exercise. J Biomech 2001;34:505-12.

25. Rasquinha $E$, Klencz CA, Mangini M. Estudo dos componentes físicos implicado no ângulo de aterrissagem no movimentos do rodante, executado no aparelho solo da Ginástica Artística. Rev Bras Med Esporte 2006;12:184-8.

26. Takei Y, Dunn JH, Beucker EP. Somersaulting techniques used in high-scoring and low-scoring roche vaults performed by male Olympic Gymnasts. J Spots Sci 2007;25:673-85. 